\title{
Policistina-1 protege a los cardiomiocitos de la necrosis inducida por estrés mecánico
}

\author{
Cristián Espinoza ${ }^{1}$, Magda C. Díaz ${ }^{1,2,3}$, Magdalena González ${ }^{1,2}$, Ivonne Olmedo ${ }^{1}$, Zully Pedrozo ${ }^{1,2,4}$. \\ 1 Instituto de Ciencias Biomédicas (ICBM), Facultad de Medicina, Universidad de Chile, Santiago, Chile. \\ 2 Advanced Center for Chronic Disease (ACCDiS), Facultad de Ciencias Químicas y Farmacéuticas \& Facultad de Medicina, Universidad de \\ Chile, Santiago, Chile. \\ 3 Grupo de Investigación en Ciencias Básicas y Clínicas de la Salud. Pontificia Universidad Javeriana de Cali, Cali, Colombia. \\ 4 Center for Studies of Exercise, Metabolism \& Cancer (CEMC), Facultad de Medicina, Universidad de Chile, Santiago, Chile.
}

Apoyo financiero: FONDECYT 1150887 y 1180613 (Z.P.); 11170962 (I.O.);

FONDAP ACCDIS 15130011.

Antecedentes: La muerte de los cardiomiocitos es determinante en el desarrollo de patologías cardiacas posteriores al infarto del miocardio y la insuficiencia cardiaca. Las variaciones en la expresión de la familia de proteínas BCL-2 regulan vías, tanto de muerte, como de sobrevida celular. Así, BCL-2 es una proteína anti- apoptótica y NIX una proteína que induce la necrosis y/o la apoptosis celular. La Policistina-1 (PC1) es un mecanosensor vital para la función contráctil cardiaca; sin embargo, se desconoce su papel en la sobrevida de los cardiomiocitos durante el estrés mecánico.

Objetivo: Determinar si PC-1 previene la muerte de los cardiomiocitos inducida por estrés mecánico y las proteínas BCL-2 y NIX.

Métodos: Se utilizó cultivo de cardiomiocitos de ratas neonatas controles o deficientes en la expresión de PC1, estimulados con solución hiposmótica (HS), como modelo de estrés mecánico. Se midió la muerte por necrosis y apoptosis y los niveles de BCL-2 y NIX. Resultados: La deficiencia de la PC1 en los cardiomiocitos induce un aumento de la necrosis y los niveles proteicos de NIX en las células estimuladas con HS. El estrés mecánico induce la apoptosis basal relacionada a una disminución de BCL- 2, independiente de la expresión de la PC1.

Conclusiones: La PC1 protege a los cardiomiocitos de la necrosis por estrés mecánico, lo que podría deberse en parte a su papel en la regulación de los niveles de las proteínas NIX.

Palabras claves: Cardiomiocito, muerte celular, estrés mecánico, Policistina-1.

\section{Correspondencia:}

Zully Pedrozo.

zpedrozo@med.uchile.cl 


\section{Policystin-1 protects cardiomyocytes from mechanical stress induced necrosis}

Background: Cardiomyocytes death is a determining factor in the development of cardiac dysfunction after myocardial infarction and heart failure. The change in BCL-2 family protein expression regulates both cell death and survival pathways, whereas BCL-2 is an anti-apoptotic protein and NIX induces necrosis and/or apoptosis. Polycystin-1 (PC1) is a crucial mechanosensor for cardiac contractile function. However, its role in cardiomyocyte survival during mechanical stress is unknown.

Aim: To study the relationship of PC1 with mechanical stretch-death in cardiomyocytes and the BCL-2, and NIX proteins.

Methods. Controls or deficient expression of PC1 neonatal rat ventricular myocytes were stimulated with hypoosmotic solution (HS) and used as a model of mechanical stress. Necrosis or apoptosis cell death, BCL-2 and NIX protein levels were measured.

Results: Deficient expression of $\mathrm{PC} 1$ increases cardiomyocyte necrosis and NIX protein levels in cells stimulated with HS. Mechanical stress induces basal apoptosis related to a decrease in BCL-2, independent of $\mathrm{PC} 1$ expression.

Conclusion: $\mathrm{PC} 1$ protects cardiomyocytes from mechanical stress necrosis, at least in part, by regulating NIX protein levels.

Keywords: cardiomyocyte, cell death, mechanical stretch, Polycystin-1. 


\section{Introducción:}

Las células musculares cardiacas o cardiomiocitos 1levan a cabo la función de contracción del corazón. En cada latido, el corazón se encuentra sometido a un proceso de estiramiento mecánico, donde las fuerzas internas de contracción y relajación, o la deformación de la membrana celular, son sensadas a través de receptores llamados mecanosensores, los que transforman la señal mecánica en química, permitiendo su integración río abajo para regular varios procesos celulares, entre ellos la sobrevida celular ${ }^{1-4}$.

Un estiramiento sostenido o mayor al fisiológico se define como estrés mecánico (EM). El EM es capaz de modular, a su vez, el crecimiento, remodelamiento y cambios en la expresión génica que derivan en variaciones en el metabolismo y la sobrevida o la muerte de los cardiomiocitos $1,3,5$. De hecho, el EM ha sido señalado como una de las primeras causas del daño y desarrollo de la hipertrofia cardiaca ${ }^{6}$ y del aumento de la muerte de los cardiomiocitos durante el infarto agudo de miocardio $(\mathrm{IAM})^{7}$, en el cual el EM es producido por el aumento del volumen celular durante la reperfusión ${ }^{8}$. Controversialmente, algunos estudios sugieren que, si el EM es desarrollado previo al IAM, este confiere protección al tejido cardiaco ${ }^{9}$. Diferentes estudios han propuesto a las integrinas, las cadherinas, los canales activados por estiramiento, la Policistina-1 y algunas proteínas G como mecanosensores en el tejido cardiaco ${ }^{1,5-6}$. Pese a la importancia que parecería tener el EM en el corazón, se desconoce a cabalidad la identidad de los mecanosensores expresados en los cardiomiocitos, al igual que las vías de señalización que éstos regulan.

Las policistinas son una familia de proteínas transmembrana cuyos principales representantes son la Policistina-1 (PC1) y la Policistina-2 (PC2). Mientras que PC2 es un canal de $\mathrm{Ca} 2+$ no específico, $\mathrm{PC} 1$ es un mecanosensor capaz de regular la función de la PC2 o de ejercer su función en forma independiente ${ }^{2,10}$. Se expresan en diferentes tejidos, entre ellos el cardiaco; sin embargo, su función ha sido caracterizada, principalmente, en los cilios primarios de las células renales ${ }^{10-11}$, donde la PC1 sensa el flujo de orina activando canales de $\mathrm{Ca} 2+{ }^{2,10}$. Se ha reportado, además, que estaría implicada en la proliferación, adhesión y diferenciación celular, pero las vías de señalización son poco claras ${ }^{12}$. A nivel cardiaco, nuestro laboratorio ha reportado que la PC1 es un mecanosensor presente en los cardiomiocitos, vital para la función contráctil del corazón y que se encuentra involucrada en el desarrollo de la hipertrofia cardiaca inducida por EM in vitro (estrés hiposmótico) e in vivo (constricción aórtica transversa) ${ }^{11}$. A pesar de que la PC1 tiene una función vital en la función cardiaca, se desconoce hasta el momento los mecanismos asociados a dicha regulación.

La muerte y pérdida de los cardiomiocitos por necrosis o apoptosis, lleva a la disminución de la función cardiaca y al remodelamiento ${ }^{13}$, siendo este un punto clave en el desarrollo de diferentes patologías post IAM, como la insuficiencia cardiaca ${ }^{13}$. En células renales, se encuentra descrito que la PC1 es clave para evitar el aumento de la muerte celular por apoptosis a través de la estabilización de los niveles proteicos de BCL-214; sin embargo, se desconoce el papel de la PC1 en la muerte de los cardiomiocitos.

BCL-2 es una familia de proteínas caracterizadas por presentar dominios de homología llamadas BCL-2. Estas proteínas tienen una función principal en la regulación de la apoptosis, ya sea a través de su promoción, por aumento de la expresión de algunos de sus miembros como BCL-2 o BCL-XL, o a través de su inhibición, por la inducción de la expresión de BAX, BAK o BCL-Xs, entre otros ${ }^{15}$. Además de lo anterior, está demostrado que un subgrupo de la familia de proteínas BCL-2, llamado "BNIP" (BNIP1, BNIP2, BNIP3, y BNIP 3L o NIX), son capaces de inducir la muerte celular, ya sea por apoptosis o necrosis ${ }^{16-17}$. Mientras BNIP3 es inducido por hipoxia y participa en la muerte celular ante isquemia cardiaca ${ }^{18-19}$, la proteína NIX parece estar más asociada con señales de hipertrofia patológica, como la inducida por sobrecarga de presión o estrés mecánico ${ }^{19-20}$.

Así, la muerte celular, la fibrosis y el remodelamiento y la falla cardíaca, se encuentran relacionados tanto a una disminución de la expresión de $\mathrm{NIX}^{20}$, como a la disminución de la expresión de BCL-2 y BCL-XL ${ }^{19}$.

De acuerdo con lo anterior, el objetivo de este trabajo fue estudiar la relación entre la presencia de la PC1 y la muerte de los cardiomiocitos durante el EM y su asociación con la expresión de BCL-2 y NIX.

\section{Materiales y Métodos}

Cultivo de miocitos ventriculares de ratas neonatas (NRVM) y transfecciones La mantención y manipulación de los animales se realizó de acuerdo con las normas éticas establecidas para animales de experimentación "Guide for the care and use of laboratory animals" (National Institutes of Health, EEUU, 2011).

Para la obtención de los NRVM se utilizaron ratas neonatas Sprague-Dawley (1 a 3 días de edad), de ambos sexos, según lo descrito previamente ${ }^{11}$. 
Para disminuir la expresión de PC1 en los NRVM, los mismos fueron transfectados durante toda la noche con un siRNA de secuencia específica (siPC1, Sigma- Aldrich, $120 \mathrm{nM}$ ), de acuerdo con lo descrito previamente ${ }^{11}$.

\section{Estrés mecánico}

Los NRVM se mantuvieron 24 horas en medio de mantención sin suero, previo al estímulo con solución hiposmótica (medio de mantención diluido 1:2), utilizada como modelo de estrés mecánico ${ }^{11,21}$, el cual se mantuvo por 48 horas.

\section{Western blot}

Las proteínas se obtuvieron desde los NRVM lisados (T-PER ${ }^{\circledR}$ Tissue Protein Extraction Reagent, Thermo Fisher Scientific), junto a inhibidores de proteasas (Complete Protease Inhibitor Cocktail Tablets EASY packs, Roche) y fosfatasas (PhosSTOP-Phosphatase inhibitor cocktail tablets, Roche). Los lisados se centrifugaron a $10.000 \mathrm{x} \mathrm{g} \mathrm{a} 4^{\circ} \mathrm{C}$ y se cuantificó la concentración de proteínas por el método de Bradford (Protein Assay Dye Reagent, Bio-Rad). Las proteínas se separaron en geles de poliacrilamida (SDS-PAGE), transfirieron a una membrana de soporte de PVDF (Difluoruro de polivinilideno, Immobilon®_psq, Merck), y expusieron a anticuerpos específicos para NIX y BCL-2 (Santa Cruz Biotechnology). Se utilizaron los niveles de GAPDH (Sigma-Aldrich), como control de carga. La cuantificación de los niveles proteicos se realizó utilizando el programa Image Lab.

\section{Cuantificación de Lactato Deshidrogenasa (LDH)}

Se utilizó el kit CytoTox96® Non-Radioactive Citotoxicity Assay (Promega) para medir los niveles de LDH liberados al medio de cultivo (proveniente de las células muertas), así como el de las células remanentes en las placas (células vivas), según indicaciones del fabricante. Los niveles de LDH del sobrenadante se expresaron como porcentaje del total de LDH de cada placa.

\section{Citometría de Flujo}

La muerte por apoptosis se evaluó a través de la detección de Anexina-V-FITC (Annexin V-FITC Apoptosis Detection Kit, Abcam). Debido a que la anexina $\mathrm{V}$ puede teñir también las células necróticas, se utilizó una co-tinción con ioduro de propidio para diferenciar las células necróticas de las apoptóticas, de acuerdo con lo recomendado por el fabricante. Las células se analizaron en un citómetro de flujo. Los datos obtenidos fueron analizados con el programa Cyflogic.

\section{Reactivos}

DMEM, M199, solución de penicilina: estreptomicina, suero fetal bovino y suero equino fueron provistos por Hyclone, GE Healthcare Life Sciences. La 5-bromo-2desoxiuridina y la gelatina fueron obtenidas de Sigma-Aldrich.

\section{Análisis estadístico}

Los resultados se muestran como el promedio \pm S.E.M. de los experimentos independientes realizados (n) y se analizaron utilizando t-test o ANOVA de una vía seguido de un pos-test de Dunnet. Las diferencias se consideraron significativas con $\mathrm{p}<0,05$.

\section{Resultados}

La expresión deficiente de PC1 aumenta la necrosis de los NRVM durante el estrés mecánico, sin cambios en la muerte por apoptosis.

Los NRVM controles y deficientes para la expresión de PC1 (siPC1), se expusieron por 48 horas a una solución hiposmótica (HS), utilizada como modelo de estrés mecánico y, posteriormente, se midieron los niveles de necrosis y apoptosis de las muestras. El estímulo de HS solo induce un aumento de la muerte por necrosis en los NRVM siPC1 (Figura 1A). Por otra parte, el estímulo con HS induce un aumento de la apoptosis celular basal, independiente de la presencia de la PC1 (Figura 1B). El conjunto de nuestros datos sugiere que la PC1 actuaría

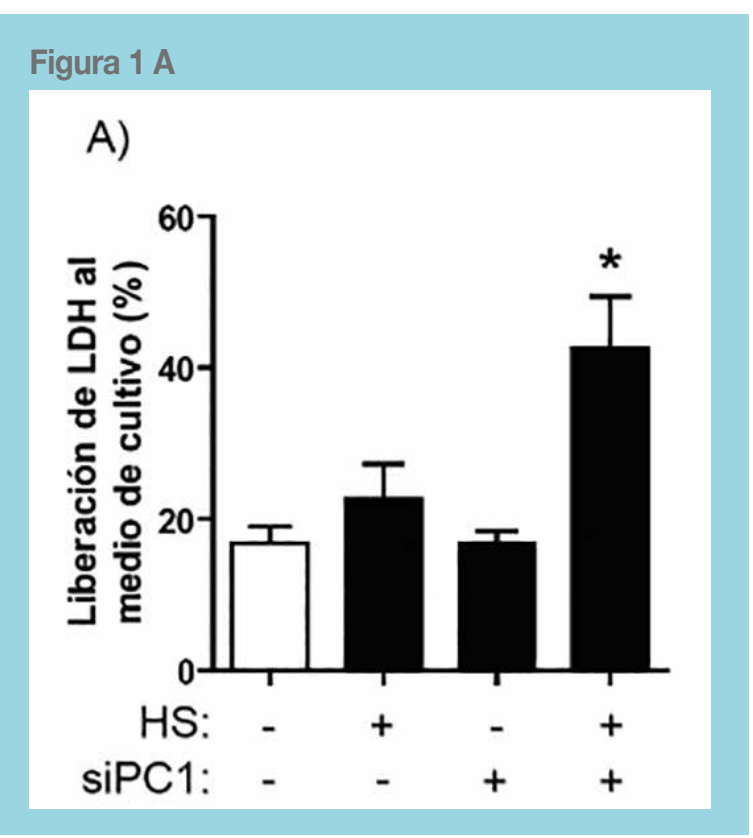




\section{Figura 1 B}

\section{B)}

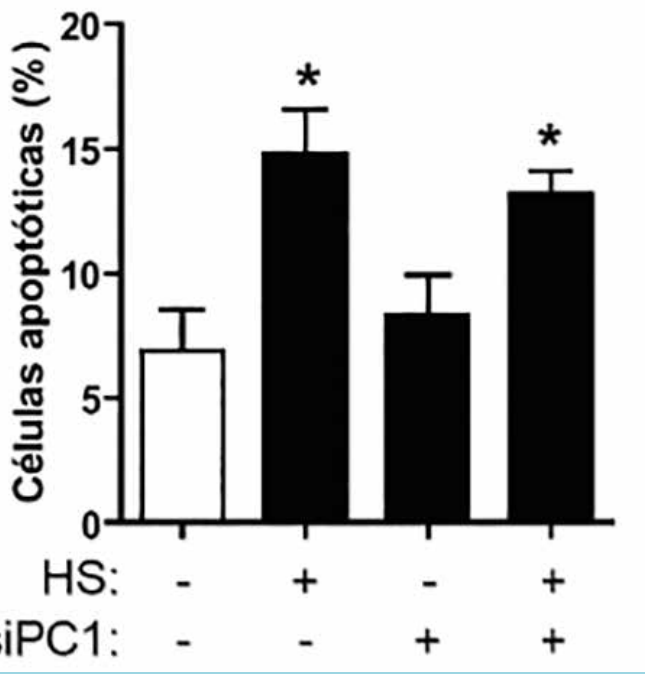

Figura 1.- Efecto de la deficiencia de la Policistina-1 en la muerte de los cardiomiocitos durante estrés mecánico. Cardiomiocitos controles y deficientes para la expresión de la PC1 (siPC1), se expusieron a solución hiposmótica (HS) como modelo de estrés mecánico y se midió: A) Liberación de lactato deshidrogenasa $(\mathrm{LDH})$ al medio de cultivo como índice de necrosis, expresado como el porcentaje de la actividad total (n=7-9) y B) Índice de células apoptóticas expresado como porcentaje ( $n=3$ en duplicado). Los valores se muestran como promedios $\pm S E M$ y fueron analizados mediante ANOVA de una vía seguido por un post-test de Dunnet. $* P<0,05$ vs. control.

evitando el aumento de la necrosis de los NRVM sometidos a estrés mecánico por HS.

El estrés mecánico induce el aumento proteico de NIX en NRVM deficientes para la expresión de PC1.

Se midieron los niveles proteicos de NIX y BLC-2 en NRVM controles y siPC1 expuestos a HS. Mientras el HS o la deficiencia de la PC1 no induce cambios en el contenido de NIX, las células siPC1 estimuladas con HS presentan un aumento de sus niveles proteicos (Figura 2A). Por otra parte, el estímulo con HS induce una disminución del contenido proteico de BCL-2, el cual es independiente de la presencia de la PC1 (Figura 2B). Además, la relación BCL-2/NIX disminuye en los NRVM estimulados con HS, independiente de la presencia de la PC1 (Figura 2C).

Estos datos en su conjunto sugieren que la presencia de la PC1 en los cardiomiocitos evita el aumento del contenido proteico de NIX durante el estrés mecánico, sin relevancia en las variaciones de la proteína anti-apoptótica BCL-2.

\section{Discusión}

El presente trabajo investigó el papel de la Policistina-1 (PC1) de los cardiomiocitos como regulador de la sobrevida celular durante el estrés mecánico. En cada diástole, las células cardiacas sufren un estiramiento mecánico dentro de límites fisiológicos, lo que activa diferentes vías de señalización relacionadas con procesos celulares, como la diferenciación y la sobrevida celular ${ }^{22}$. Sin embargo,

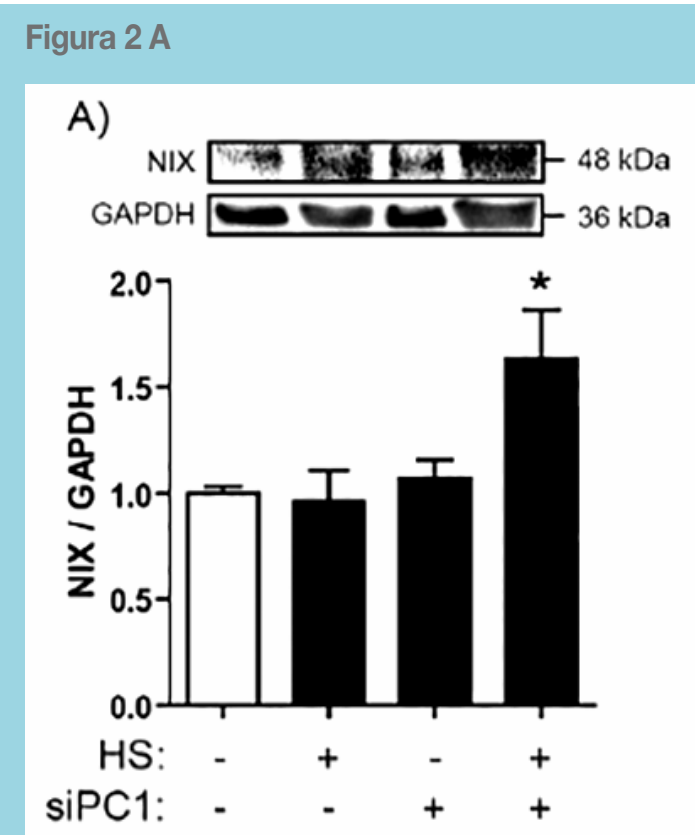

Figura 2 B

B)
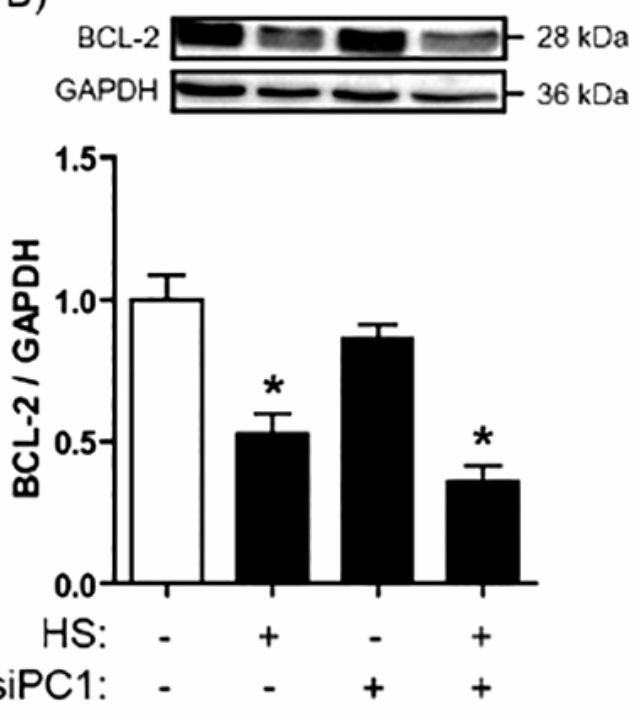


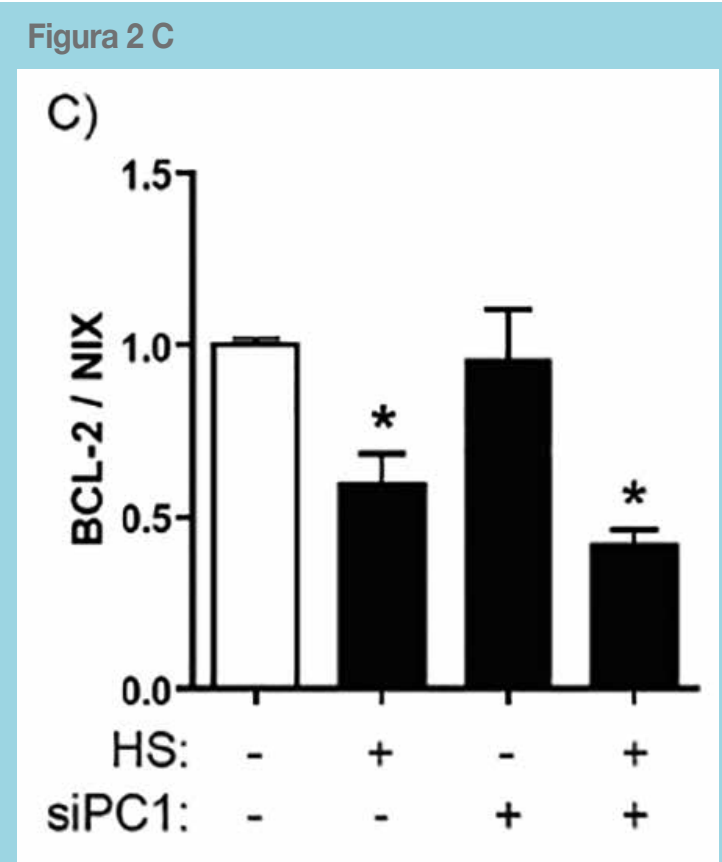

Figura 2.- Cambios en los niveles proteicos de NIX y BCL-2 en cardiomiocitos deficientes para la expresión de la Policistina-1 (siPC1) expuestos a solución hiposmótica (HS). En la parte superior se observan western blots representativos para NIX (A) y BCL-2 (B). GAPDH se utilizó como control de carga. En la parte inferior se muestran los gráficos de barras correspondientes, expresando la relación NIX/GAPDH para cada situación $(n=5-6)$. En C) se muestra la relación BCL-2/NIX. Los valores se muestran como promedio $\pm S E M$, analizados mediante ANOVA de una vía seguido por un post-test de Dunnet. ${ }^{*} P<0,05$ vs. control.

cuando el estiramiento supera los límites fisiológicos se produce un estrés mecánico que activa vías de señalización diferentes a las anteriores, que puede llevar a cambios en el metabolismo o la expresión de genes y conducir a la muerte celular por necrosis o apoptosis $22-23$.

Reportes previos de nuestro laboratorio indican que la PC1 es un mecanosensor presente en el cardiomiocito, vital para el funcionamiento contráctil del miocardio ${ }^{11}$; sin embargo, se desconocen los mecanismos involucrados en dicha función o el papel que dicho mecanosensor podría tener durante el estrés mecánico de los cardiomiocitos.

Nuestros resultados muestran que la presencia de la PC1 en el cardiomiocito, estimulado con solución hiposmótica (HS) como modelo de estrés mecánico, evita la muerte por necrosis, sin que su expresión deficiente altere los niveles basales de muerte celular. Por otro lado, los datos sugieren que la $\mathrm{PC} 1$ no tiene un papel protector en la muerte por apoptosis inducida por la HS. Estos resultados están en concordancia con estudios realizados en células renales, donde la PC1 actúa como un protector de la muerte por necrosis inducida durante estímulos de isquemia/reperfusión ${ }^{24}$. Sin embargo, mientras que en dichos estudios la deficiencia de PC1 también se encuentra relacionada a un aumento de la apoptosis, al igual que en otros reportes, en nuestro modelo la apoptosis es independiente de la $\mathrm{PC} 1^{24-25}$. Estos resultados discordantes podrían deberse a la diferencia del tipo celular o al estímulo utilizado en los estudios, así como a limitaciones técnicas para la medición de apoptosis Si bien realizamos una co-tinción para diferenciar células apoptóticas de necróticas, otras técnicas deberían utilizarse para validar los resultados encontrados.

Debido a que los cardiomiocitos son células terminalmente diferenciadas, con poca o nula capacidad proliferativa, la muerte de estos redunda en el desarrollo de diferentes patologías cardiacas ${ }^{4}$. Nuestros resultados sugieren entonces a la PC1 como una proteína importante para prevenir el aumento del daño del tejido cardiaco, debido a la muerte de los cardiomiocitos.

Además de la clasificación clásica de muerte celular en apoptosis, autofagia y necrosis, actualmente se reconocen otros tipos de muerte centradas en los aspectos moleculares del proceso, como por ejemplo, la necrosis regulada o programada, la cual es iniciada por alteraciones específicas del microambiente intracelular, como el estrés oxidativo y la sobrecarga citosólica de $\mathrm{Ca} 2+26$. En este tipo de muerte, la activación de una o más vías de señalización conducen frecuentemente a la apertura del poro de transición de permeabilidad mitocondrial (MPTP), que interrumpe la función respiratoria mitocondrial y desencadena finalmente la necrosis ${ }^{26}$.

En cuanto al mecanismo que subyace a la muerte evidenciada, el estrés mecánico induce el aumento proteico de NIX cuando los cardiomiocitos son deficientes en la expresión de PC1. Debido a que NIX es una proteína involucrada, tanto en el aumento de la muerte por necrosis programada, como por apoptosis 17,27 , nuestros datos sugieren que NIX podría ser parte de la vía responsable de activar la necrosis de los cardiomiocitos bajo estas condiciones. Así, en condiciones de estrés mecánico, la PC1 estaría inhibiendo el aumento de NIX y evitando el aumento de la muerte inducida por el estrés mecánico. Futuros estudios son necesarios para corroborar el tipo de necrosis (programada o no programada) en el cual está implicado el rol protector de la PC1 y la importancia de la vía que involucra a Nix en este proceso.

Si bien BCL-2 ha sido descrita, principalmente, como anti-apoptótica, en los últimos tiempos se la ha descrito como vía común para la inhibición de diferentes tipos de 
muerte, como el de la autofagia y el de la necrosis programada $^{28}$. Aun cuando en trabajos previos realizados en células renales se muestra que la ausencia de la PC1 induce la degradación de BCL-2, originando la apoptosis ${ }^{25}$, en nuestro modelo el estrés mecánico reduce el contenido proteico de BCL-2 en los cardiomiocitos de manera independiente a la expresión de la PC1. Así, es posible que el estrés mecánico induzca más de un tipo de muerte en los cardiomiocitos y que la PC1 esté implicada en la protección de sólo algunos de ellos, pero no de todos, como el de la apoptosis y que la disminución de BCL-2 esté más bien ligada a este tipo de muerte celular. Se requieren experimentos complementarios para definir el papel de la PC1 en la muerte por necrosis programada y la implicancia de BCL-2 en la misma en nuestro modelo.
El conjunto de nuestros hallazgos indica, por primera vez, que la PC1 es una proteína protectora ante la necrosis de los cardiomiocitos inducida por el estrés mecánico. El mecanismo implicaría, aunque sea en parte, la inhibición del aumento del contenido proteico de la proteína NIX. Futuros experimentos son necesarios para confirmar el mecanismo a través del cual la PC1 es capaz de regular la expresión de esta proteína.

\section{Agradecimientos:}

Este trabajo fue financiado por el Fondo Nacional de Investigación Científica y Tecnológica, FONDECYT (1150887 y 1180613 para Z.P.; 11170962 para I.O.); FONDAP (ACCDiS 15130011 para Z.P.) y CEMC (para Z.P.).

\section{Referencias}

1. LAMMERDING J, KAMM RD, LEE RT. Mechanotransduction in cardiac myocytes. Ann N Y Acad Sci. 2004; 1015: 5370.

2. RETAILLEAU K, DUPRAT F. Polycystins and partners: proposed role in mechanosensitivity. J Physiol. 2014; 592: 24532471.

3. SHYU KG. Cellular and molecular effects of mechanical stretch on vascular cells and cardiac myocytes. Clin Sci. 2009; 116: $377-389$.

4. XIN M, OLSON EN, BASSEL-DUBY R. Mending broken hearts: cardiac development as a basis for adult heart regeneration and repair. Nat Rev Mol Cell Bio. 2013; 14: 529-541.

5. McCAIN ML, PARKER KK. Mechanotransduction: the role of mechanical stress, myocyte shape, and cytoskeletal architecture on cardiac function. Pflugers Arch. 2011; 462: 89-104.

6. RUWHOF C, VAN DER LAARSE A. Mechanical stress-induced cardiac hypertrophy: mechanisms and signal transduction pathways. Cardiovasc Res. 2000; 47: 23-37.

7. MOZAFFARI MS, LIU JY, ABEBE W, BABAN B. Mecha- nisms of load dependency of myocardial ischemia reperfusion injury. Am J Cardiovasc Dis. 2013; 3: 180-196.

8. GARCIA-DORADO D, ANDRES-VILLARREAL M, RUIZ-MEANA M, INSERTE J, BARBA I. Myocardial edema: a translational view. J Mol Cell Cardiol. 2012; 52: 931-939.

9. HAO J, KIM HS, CHOI W, HA TS, AHN HY, KIM CH. Mechanical Stretch- Induced Protection against Myocardial Ischemia-Reperfusion Injury Involves AMP- Activated Protein Kinase. Korean J Physiol Pharmacol. 2010; 14: 1-9.

10. CHAPIN HC, CAPLAN MJ. The cell biology of polycystic kidney disease. J Cell Biol. 2010; 191: 701-710.

11. PEDROZO Z, CRIOLLO A, BATTIPROLU PK, MORALES CR, CONTRERAS- FERRAT A, FERNÁNDEZ C, et al. Polycystin-1 is a cardiomyocyte mechanosensor that governs L-type Ca2+ channel protein stability. Circulation. 2015; 131: 2131-2142.

12. DALAGIORGOU G, BASDRA EF, PAPAVASSILIOU AG. Polycystin-1: function as a mechanosensor. J Biochem Cell Biol. 2010; 42: 1610-1613. 
13. CHIONG M, WANG ZV, PEDROZO Z, CAO DJ, TRONCOSO R, IBACACHE M, et al. Cardiomyocyte death: mechanisms and translational implications. Cell Death Dis. 2011; 2: e244.

14. YU W, RITCHIE BJ, SU X, ZHOU J, MEIGS TE, DENKER BM. Identification of polycystin-1 and Ga12 binding regions necessary for regulation of apoptosis. Cell Signal. 2011; 23: 213-221.

15. AN J, LV J, LI A, QIAO J, FANG L, LI Z, et al. Constitutive expression of Bcl-2 induces epithelial-Mesenchymal transition in mammary epithelial cells. BMC Cancer. 2015; 15: 476-483.

16. CHEN Y, LEWIS W, DIWAN A, CHENG EHY, MATKOVICH SJ, DORN GW. Dual autonomous mitochondrial cell death pathways are activated by Nix/BNip3L and induce cardiomyopathy. Proc Natl Acad Sci USA. 2010; 107: 90359042 .

17. KUNG G, KONSTANTINIDIS K, KITSIS RN. Programmed necrosis, not apoptosis, in the heart. Circ Res. 2011; 108: 10171036.

18. KUBASIAK LA, HERNANDEZ OM, BISHOPRIC NH, WEBSTER KA. Hypoxia and acidosis activate cardiac myocyte death through the Bcl-2 family protein BNIP3. Proc Natl Acad Sci USA. 2002; 99: 12825-12830.

19. ZHANG J, NEY PA. Role of BINP3 and NIX in cell death, autophagy, and mitophagy. Cell Death Differ. 2009; 16: 939-946.

20. DIWAN A, WANSAPURA J, SYED FM, MATKOVICH SJ, LORENZ JN, DORN GW. Nix-mediated apoptosis links myocardial fibrosis, cardiac remodeling, and hypertrophy decompensation. Circulation. 2008; 117: 396-404.

21. TANG W, STRACHAN RT, LEFKOWITZ RJ, ROCKMAN
HA. Allosteric modulation of $\beta$-arrestin-biased angiotensin II type 1 receptor signaling by membrane stretch. J Biol Chem. 2014; 289: 28271-28283.

22. KNÖLL R, HOSHIJIMA M, HOFFMAN HM, PERSON V, LORENZEN- SCHMIDT I, BANG ML, et al. The cardiac mechanical stretch sensor machinery involves a $\mathrm{Z}$ disc complex that is defective in a subset of human dilated cardiomyopathy. Cell. 2002; 111: 943-955.

23. YASUKAWA H, HOSHIJIMA M, GU Y, NAKAMURA T, PRADERVAND S, HANADA T, et al. Suppressor of cytokine signaling-3 is a biomechanical stress- inducible gene that suppresses gp130-mediated cardiac myocyte hypertrophy and survival pathways. J Clin Invest. 2001; 108: 1459-1467.

24. BASTOS A, PIONTEK K, SILVA AM, MARTINI D, MENEZES LF, FONSECA JM, et al. Pkd1 haploinsufficiency increases renal damage and induces microcyst formation following ischemia/reperfusion. J Am Soc Nephrol. 2009; 20: 2389-2402.

25. YU W, KONG T, BEAUDRY S, TRAN M, NEGORO H, YAMADALA V, et al. Polycystin-1 protein level determines activity of the Ga12/JNK apoptosis pathway. J Biol Chem. 2010; 285: 10243-10251.

26. GALLUZZI L, VITALE I, AARONSON SA, ABRAMS JM, ADAM D, AGOSTINIS P et al. Molecular mechanisms of cell death: recommendations of the Nomenclature Committee on Cell Death 2018. Cell Death Differ. 2018; 25: 486- 541.

27. DORN GW 2nd. Nix Nought Nothing: fairy tale or real deal. J Mol Cell Cardiol. 2011; 51: 497-500.

28. SASI N, HWANG M, JABOIN J, CSIKI I, LU B. Regulated cell death pathways: new twists in modulation of BCL2 family function. Mol Cancer Ther. 2009; 8: 1421- 1429. 